\title{
One in 18 patients harmed in hospital
}

$\mathrm{O}$ ne in 18 patients admitted to hospital in Canada suffers avoidable harm during their stay, reports the Canadian Institute for Health Information (CIHI).

Patients experienced preventable harm that was serious enough to require treatment or a longer hospital stay in 138000 cases, or 5.6\% of hospitalizations in 2014-15, according to the benchmark study Measuring Patient Harm in Canadian Hospitals. One in five of these patients suffered multiple mishaps. One in eight died in hospital.

"It's everyone's responsibility to learn as best they can from these events and work to reduce potential for harm," says Kathleen Morris, vice-president of research and analysis at CIHI. Tracking and reporting harmful events at a national level is a major step toward safer care, she adds. "Before now, we haven't had a standard way to measure and monitor the harm experienced by hospital patients."

The report includes data on 31 types of potentially preventable harm, ranging from sepsis to surgical errors. Even with a bigger lens on the issue, Morris says CIHI likely hasn't captured the full picture of harm that is happening in hospitals. For example, "data from Quebec and for select mental health patients are not included," she says.

Whatever the size of the problem, it's clear the costs are considerable. On average, patients who experienced harm spent about four extra days in hospital. "That may not seem like much, but when it's put all together it results in more than half-a-million additional hospital days," Morris explains.

Put another way, on any given day more than 1600 hospital beds, the equivalent of four large hospitals, are occupied by patients who suffered harm that extended their stays. CIHI estimates this additional care costs the health system \$685 million in 2014-15, not including

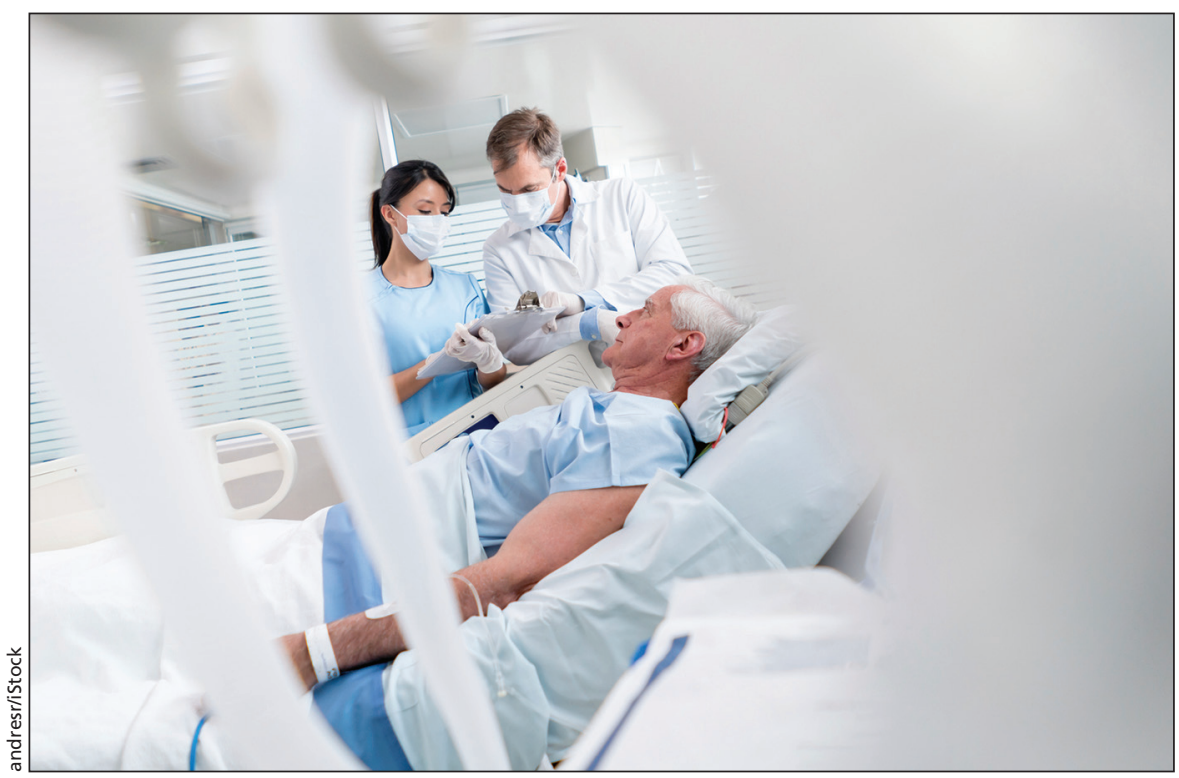

The Canadian Institute for Health Information released a benchmark study of avoidable harm in hospitals.

payments to physicians for their services. "Reducing harmful events could free up resources for the unmet needs of other patients," Morris says.

CIHI's findings suggest there are potential gains to be made by taking patient-safety initiatives back to basics. In the past decade, patient-safety initiatives have often focused on "never events," or harms that should never happen like leaving a sponge inside a surgical patient. "What's interesting about the list of the 31 we have is that the "never events' are a very small proportion of that," says Tracy Johnson, CIHI director of health system analysis and emerging issues.

The top three types of harm are electrolyte and fluid imbalance, urinary tract infections and delirium. These account for more than a third of incidents. "There's a fair amount of opportunity" for improvement in these more routine areas, Johnson says.

The report also highlighted room for improvement in care for complex patients. These patients, who had multiple conditions and multiple special- ists involved in their care, were more likely to suffer harm in hospitals. One in five of the most complex patients experienced at least one harmful event. They were also three to four times more likely to experience multiple harmful events compared to less complex patients.

The Canadian Patient Safety Institute is compiling an online library of resources to guide hospitals' improvement efforts. The new Hospital Harm Improvement Resource will provide tools, summarize best practices and suggest goals for reducing each of the 31 types of harm that CIHI is tracking. It will also provide tips for using CIHI data to improve care, information on related accreditation standards, and stories about patients and successful initiatives.

$\mathrm{CIHI}$ is also considering creating a facility-level report that hospitals could use to track their individual progress on reducing preventable harms. - Lauren Vogel, CMAJ

CMAJ 2016. DOI:10.1503/cmaj.109-5351 